\title{
On the Performance of Alternating Concurrent
}

\section{Cooperative Transmissions in the High}

\section{Path-Loss Attenuation Regime}

\author{
Aravind Kailas \\ Dept. of Electrical and Computer Engineering \\ The University of North Carolina at Charlotte \\ Charlotte, NC 28223, USA \\ Tel: 1-608-628-1443Ｅ-mail: aravindk@ieee.org
}

Received: March 20, 2012

DOI: $10.5296 /$ npa.v4i2.1500
Accepted: May 21, 2012 Published: June 19, 2012

URL: http://dx.doi.org/10.5296/ npa.v4i2.1500

\begin{abstract}
This paper addresses the issue of network broadcasting using alternating concurrent cooperative transmissions for sensor-based wireless networks that are very lossy. The medium access control (MAC)-free broadcast strategy is a simple, energy-efficient, low-overhead form of cooperative diversity-based strategy called the opportunistic large arrays (OLAs), and uses a received power-based threshold to define mutually exclusive sets of nodes during the initial broadcast, such that the union of the sets includes all the nodes in the network or cooperative route. This eliminates the formation of undesirable network coverage holes, a result of the "dead" nodes resulting from repeated usage. This broadcast strategy has been analyzed for wireless channels with $\gamma=2$. The semi-analytical approach presented here investigates network life extensions for $\checkmark>2$ by considering two extreme continuum network topologies that correspond to the largest and smallest ratios of nodes (or network areas) used up during a successful broadcast, namely discs and strips, analyzing which will then set the bounds for arbitrary shaped static, cooperative routes or networks.
\end{abstract}

Keywords: Broadcast, cooperative diversity, energy efficiency, opportunistic large arrays, sensor networks 


\section{Motivation}

Body area networks (BANs) and short-range indoor links are examples of very lossy communication media, where the path loss exponents range between 3 and 7, a lot different from the free space propagation exponent (i.e., $\gamma=2$ ) [1]. The high path loss impacts the energy consumption, and subsequently, the network operation lives. At higher path losses, cooperative diversity-based approaches become advantageous and sometimes an absolute requirement to maintain the connectivity of the system. Cooperative transmission (CT)-based strategies leverage the spatial diversity in a network by having multiple nodes transmit the same message, and offer an signal-to-noise ratio (SNR) advantage in a multipath fading environment that could be used to lower total transmission power expended in the network, achieve range extension, etc., [2], [3]. The energy consumption can be decreased in such harsh conditions because the transmission effort is spread over the whole network. This correspondence considers a simple, low-overhead form of cooperative diversity called the opportunistic large arrays (OLAs), wherein a group of nodes autonomously fire at approximately the same time the transmit diversity waveforms in response to energy received from a single source or another OLA [4], which will be referred to as "Basic OLA." OLA with a transmission threshold (OLA-T), an energy-efficient version of Basic OLA, applies a received signal power-based threshold to recruit cooperators at the edge of the decoding range [5], [6]. The alternating OLA-T (A-OLA-T), a non-trivial extension of OLA-T, overcomes the formation of dead zones in a network by avoiding using the same sets of cooperating nodes during successive broadcasts [7]. Sec. I-A provides a very brief review of the OLA-based protocols for the benefit of the reader not familiar with this family of cooperative broadcasting protocols.

Contributions: The overriding purpose of this correspondence is to investigate the impact of A-OLA-T broadcasting in wireless channels with higher path-attentuation. The necessary and sufficient conditions for a successful broadcast using A-OLA-T have been derived for a deterministic path loss model with a path loss exponent of 2. Being favorably-placed, the border nodes would be expected to dominate the OLA transmission energy even more as the wireless channel becomes more lossy (i.e., the path loss exponent increases), thereby widening the gap in the energy consumption between Basic OLA and OLA-T, and hence, the network lives 1 between Basic OLA and A-OLA-T. With this in mind, this correspondence investigates the performance of A-OLA-T in environments with higher $(>2)$ path loss exponents. Bounds on the network life extensions offered by A-OLA-T relative to Basic OLA as a function of key network parameters at higher path loss exponents have been derived semi-analytically for continuum and finite density networks, and are the original contributions of this correspondence. Two contrasting and extreme network topologies corresponding to the largest and smallest ratios of nodes (or areas) used up during a successful broadcast are considered for the analysis to set the performance bounds for arbitrary-shaped cooperative routes or networks.

\subsection{Background of OLA-Based Broadcast Protocols}

The OLA, as mentioned previously, is a simple form of CT wherein a group of nodes 
autonomously fire the transmit diversity waveforms at approximately the same time, in response to energy received from a single source or another OLA [4]. Recently, the authors of [8] have demonstrated transmission time synchronization with the root mean square transmit time delay spreads less than $100 \mathrm{~ns}$ for OLAs. One simple, power amplifier-friendly way to achieve transmit diversity is to transmit on-off-shift keying (OOK) or frequency-shift keying (FSK) on orthogonal carriers, with simple energy detectors in the receiver [8]. The total transmission energy in the wireless system can still be saved despite many nodes participating in an OLA transmission because all nodes can lower their transmit powers dramatically, implying that large fade margins are not needed.

\subsubsection{Basic OLA}

We will refer to the first OLA-based broadcasting scheme proposed by the authors of [4] as the 'Basic OLA.' In a Basic OLA broadcast [4], a node relays immediately if it can decode and if it has not relayed before. The aim is to succeed in broadcasting the message over the whole network. The source node transmits a message and the group of neighboring nodes that receive and decode the message form Decoding Level $1\left(\mathrm{DL}_{1}\right)$, which is the disc enclosed by the smallest circle in Fig. 1(a). Next, each node in DL1 transmits the message. These transmitting nodes in $\mathrm{DL}_{1}$ constitute the first OLA. Next, nodes outside $\mathrm{DL}_{1}$ receive the superposition of relayed copies of the message. Nodes in this group that can decode the message constitute $\mathrm{DL}_{2}$, which is represented as the ring between $\mathrm{DL}_{1}$ and the next bigger concentric circle in Fig. 1(a). All the nodes in a decoding level form an OLA, which in turn generates the next decoding level. Thus, the resulting OLAs form concentric rings around the originating node. Figure 1(a) illustrates this phenomenon for a given network area (defined in Fig. 1 by the dashed line).

\subsubsection{OLA with Transmission Threshold (OLA-T)}

Figure 1(b) illustrates a successful network broadcasting scenario using OLA-T. The gray strips in Fig. 1(b) represent OLAs within each decoding level. Unlike the approach depicted in Fig. 1(a), the nodes that compose an OLA are only a subset of the nodes in a decoding level. OLA-T applies a received signal power-based threshold to form an OLA comprising the nodes at the edge of the decoding range [5], [6].

\subsubsection{Alternating OLA with Transmission Threshold (A-OLA-T)}

For a fixed source in a static network, repeated use of the same sets of nodes in every broadcast drain their batteries first, resulting in network holes. A-OLA-T, a simple, decentralized algorithm was proposed in [7] to avert this situation by using the transmission threshold to divide all the nodes in the network into many mutually exclusive sets of OLAs. For example, in the two-set A-OLA-T, the network is divided into two mutually exclusive sets as shown in Fig. 1(c). The first set is used in the initial OLA-T broadcast, while the second set is used in the second broadcast. The batteries are uniformly drained across the network because each succeeding broadcast alternates between the two sets. 


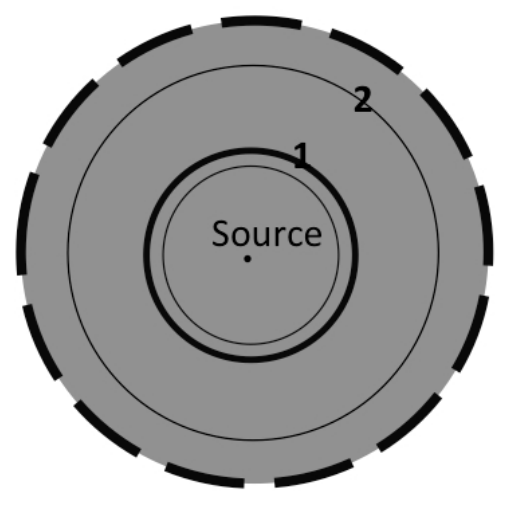

(a)

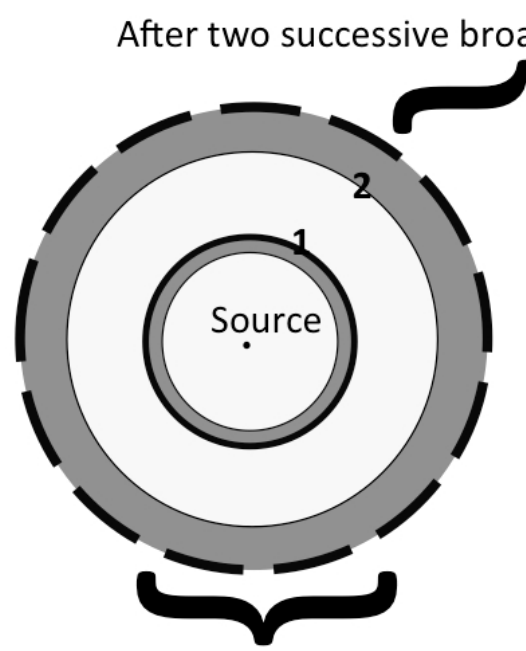

Single broadcast (OLA-T)

(b)

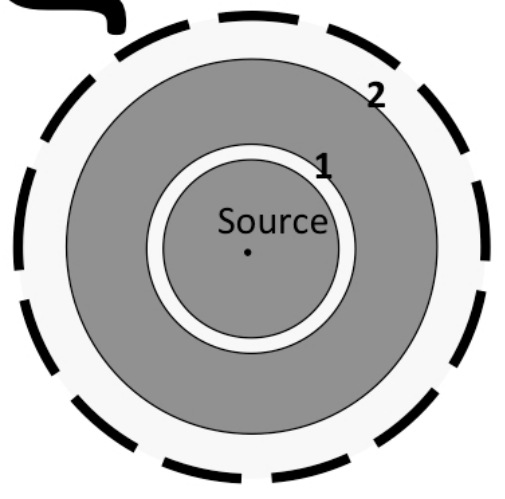

(c)

Figure 1. (a) Illustration of a successful broadcast using Basic OLA, (b) OLA with transmission threshold

(OLA-T), and (c) Alternating OLA with transmission threshold. Only nodes in the gray areas relay.

\subsection{Related Work}

In [10]-[12], the approach of dividing the nodes in non-cooperative wireless networks into disjoint sets and activating them sequentially, one set at a time to carry out network functions (e.g., monitoring, target tracking, etc.). In [10]-[11], the network is divided into sets with the objective of maximizing the coverage (in terms of sensing area or targets-tracking) of each set, and the centralized algorithms activate these sets, one at a time, using only the sensors from the current active set for monitoring all targets and for transmitting the collected data to a sink. More recently, [13] explored combining and alternating between two non-cooperative routes (formed using multipath routing and spanning tree algorithms) to prolong the operation life of nodes in a sensor network. Compared to the aforementioned non-cooperative strategies, in A-OLA-T, the sets are formed proactively based on the received signal power.

\section{Analytical Framework}

This section describes the topologies used to analyze A-OLA-T at higher path loss exponents, and to derive performance bounds and conditions for sustained operation. In order to determine the A-OLA-T performance bounds for arbitrary-shaped routes or networks, A-OLA-T broadcasts have been analyzed for the disc- and strip-shaped cooperative routes (networks), which correspond to the largest and smallest ratios of nodes (or areas) used up, respectively.

\subsection{Two-Dimensional Disc}


Half-duplex nodes are assumed. For the purpose of analysis, the nodes are assumed to be distributed uniformly and randomly over a continuous area with average density $\rho$. Again, to simply analysis, it is assumed that the originating node is a point source, and at the center of a given network area. Decode-and-forward nodes are considered such that a message can be decoded and forwarded without error when its received SNR is greater than or equal to a modulation-dependent threshold [14]. Assuming unit noise variance implies that the SNR threshold boils down to a received power criterion, which is denoted as the decoding threshold $\tau_{1}$. It should be noted that the decoding threshold $\tau_{1}$ is not explicitly used in real receiver operations such as decoding a message. If no errors are detected, then it is assumed that the receiver power must have exceeded $\tau_{1}$.

In contrast, the OLA-T uses a "user-defined" transmission or 'upper' threshold, $\tau \mathrm{u}$ that is explicitly compared to an estimate of the received SNR. This additional threshold limits the number of nodes that relay in each hop because a node would "opportunistically" relay only if its received SNR is less than $\tau \mathrm{u}$. So the thresholds, $\tau_{1}$ and $\tau_{\mathrm{u}}$, define a range of received powers that correspond to the "significant" boundary nodes, which form the OLA. We define the relative transmission threshold (RTT), $R$, as the ratio between the two thresholds. Mathematically, $R=\frac{\tau_{u}}{\tau_{l}}$. It is noted that $R>1$. Owing to the favorable location of the boundary nodes, despite transmitting at a somewhat higher power, compared to Basic OLA, there is still an overall transmit energy savings with OLA-T.

For simplicity of analysis, the deterministic model of [14] is assumed, wherein the received power at a node is equal to sum of the powers from each of the node transmissions, implying that orthogonality between the different transmitting nodes. As long as the receivers can extract the multipath diversity from the wireless channel, techniques such as transmitting on orthogonal carriers (frequency division multiplexing) or by randomly delaying the firing times (such as in [15]) will work.

Let the normalized source and relay transmit powers be denoted by $P_{s}$ and $P_{r}$, respectively, and the relay transmit power per unit area be denoted by $\overline{P_{r}}=\rho P_{r}$. The normalization is such that $P_{s}$ and $P_{r}$ are actually the SNRs at a receiver d0 away from the transmitter [5]. Since we assume a continuum of nodes in the network, we let the node density $\rho$,become very large $\rho \rightarrow \infty$, while $\overline{P_{r}}$ is kept fixed. The results given in this 
paper (in Section IV) are parameterized in terms of $R$ and node degree, $K$, which is the average number of nodes in the decoding range of a transmitter. Mathematically, for any finite node density, $K=\frac{\pi \bar{P}_{r}}{\tau_{l}}$.

\subsection{Two-Dimensional Strip}

The notation and assumptions of [5] are adopted, some of which were used earlier in [14]. Half-duplex nodes are assumed to be distributed randomly and uniformly over a continuous strip defined by $S=\left\{(x, y):|y| \leq \frac{W}{2}, 0 \leq x \leq L\right\}$ with average node density $\rho$, width $W$, and length $L$. The originating source (assumed to be a point source) and the destination are assumed to be at the opposite ends of the network strip. An OLA-based protocol may be viewed as a broadcast strategy if the entire network has a strip shape, or it may be viewed as a unicast strategy if there is a set of pre-designated cooperators along a conventional multi-hop route, which is referred to as a cooperative route. The other key system parameters such as node degree, $K$ and $R$, to name a couple, are the same as that of a two-dimensional disc that is described in Section II-A.

\subsection{Ensuring Successful Broadcasts Using Basic OLA and OLA-T}

From [14], the necessary and sufficient condition for the relayed signal to propagate in a sustained manner by concentric OLAs using the Basic OLA protocol is given by

$$
2 \geq \exp \left(\frac{1}{K}\right)
$$

From [5], it is learned that if $K$ and $R$ are constant throughout the network, they must satisfy a necessary and sufficient condition to achieve infinite network broadcast,

$$
2 \geq \exp \left(\frac{1}{K}\right)+\exp \left(\frac{-R}{K}\right) .
$$

It can be inferred that when $R \rightarrow \infty$, OLA-T becomes Basic OLA given by (1). From (2), it is observed that $K$ must approach infinity as $R \rightarrow 1$ (i.e., as $\tau_{u} \rightarrow \tau_{l}$ ), in order to maintain successful broadcast. Finally, (2) can be rewritten in terms of a lower bound for R as follows:

$$
R_{\text {lowerbound }}=-K \ln \left[2-\exp \left(\frac{1}{K}\right)\right] \text {. }
$$




\subsection{Demystifying the Normalization of Parameters}

The results given in this paper (in Section IV) have been in terms of normalized units. This section presents some examples of un-normalized values for these variables to give an idea of what power levels and node densities can achieve the various values of $K$. Expanded in terms of un-normalized variables, $K$ can be written as

$$
K=\frac{\left[\frac{P_{t} G_{t} G_{r}}{\sigma_{n}^{2}}\left(\frac{\lambda}{4 \pi d_{0}}\right)^{2}\right]\left[\frac{(\# \text { nodes }) d_{0}^{2}}{\text { Area }}\right]}{\left(\frac{\text { Rx.sensitivity }}{\sigma_{n}^{2}}\right)}=\frac{P_{t}\left[\frac{(\# \text { nodes }) d_{0}^{2}}{\text { Area }}\right]}{(\text { Rx.sensitivity }) 10^{4}},
$$

where area is normalized by the square of the reference distance, $d_{0}^{2}, P_{t}$, is the relay transmit power in $\mathrm{mW}, G_{t}$ and $G_{r}$ are the transmit and receive antenna gains, $\sigma_{n}^{2}$ is the thermal noise power, $\lambda$ is the wavelength in meters, and $\mathrm{d} 0$ is the reference distance in meters. Suppose that the radio frequency is $2.4 \mathrm{GHz}(\lambda=0.125 \mathrm{~m})$, and the antennas are isotropic $\left(G_{t}=G_{r}=\right.$ 1). Table I shows four different examples of un-normalized variables for the two-dimensional disc and strip-shaped networks. For discs, it is observed that $\mathrm{K}=7$ can be obtained in Examples 2 and 3 ranging from high density $\left(2.65\right.$ nodes $\left./ \mathrm{m}^{2}\right)$ to low density $(9$ nodes $/ 3.60$ $\mathrm{km}^{2}$ ). It is also observed that the high density cases, Examples 3 (for disc) and 4 (for strip), correspond to very low transmit powers.

Table 1. Examples of Un-Normalized Variables for a Two-Dimensional Dics- and Strip-Shaped Networks (or Routes).

\begin{tabular}{|cccc|c|c|}
\hline $\begin{array}{c}\text { Ex. } \\
\text { (dBm) }\end{array}$ & $\begin{array}{c}P_{t} \\
\text { (nodes/area) }\end{array}$ & $\begin{array}{c}\mathcal{R} \\
(\mathrm{dBm})\end{array}$ & $\mathcal{K}$ \\
\hline \hline \multicolumn{5}{|c|}{ Two-dimensional disc network } \\
\hline \hline 1 & -43.98 & 1 node $/ 4 \mathrm{~m}^{2}$ & -90.00 & 4 & 1.2595 \\
\hline 2 & -56.00 & 2.65 nodes $/ \mathrm{m}^{2}$ & -94.77 & 7 & 0.6709 \\
\hline 3 & -20.97 & 9 nodes $/ 3.60 \mathrm{~km}^{2}$ & -90.00 & 7 & 0.6709 \\
\hline 4 & -56.00 & 2.65 nodes $/ \mathrm{m}^{2}$ & -90.00 & 2 & 3.2063 \\
\hline \hline \multicolumn{7}{|c|}{ Two-dimensional strip network } \\
\hline \hline 1 & -48.00 & 3 nodes $/ \mathrm{m}^{2}$ & -90.00 & 12.56 & 0.3605 \\
\hline 2 & -48.00 & 18 nodes $/ \mathrm{m}^{2}$ & -94.77 & 2 & 3.2063 \\
\hline 3 & -56.00 & 10 node $/ \mathrm{m}^{2}$ & -90.00 & 7.85 & 0.5928 \\
\hline 4 & -20.97 & 2.5 nodes $/ \mathrm{km}^{2}$ & -90.00 & 7 & 0.6709 \\
\hline
\end{tabular}




\section{A-OLA-T Performance at Higher Path Loss Exponents}

It is noted that the concentric ring structure of the OLA propagation is still preserved owing the deterministic path loss model assumption for analysis. The outer and inner boundary radii for the $k$-th OLA ring are denoted as $r_{o, k}$ and $r_{i, k}$, respectively. Realizing that every broadcast in A-OLA-T is an OLA-T broadcast, the radiated energy consumed in a single broadcast in the first $L$ levels for a continuum case is mathematically expressed, in energy units, as $E_{\operatorname{rad}(\text { OT })}=\bar{P}_{r(O T, \min )} T_{s} \sum_{k=1}^{L} \pi\left(r_{o, k}^{2}-r_{i, k}^{2}\right)$, where $T_{s}$ is the length of the message in time units and $P_{r(\text { OT,min })}$ is the lowest value of $\bar{P}_{r}$ that is sufficient for a successful OLA-T broadcast. The energy consumed by Basic OLA is given by $E_{\text {rad (OT })}=\bar{P}_{r(O, \min )} T_{s} \pi r_{o, L}^{2}$, where $\bar{P}_{r(O, \min )}$ is the lowest value of $\bar{P}_{r}$ that would guarantee successful broadcast using Basic OLA. Because of the continuum assumption, the fraction of network life extension (FLE) for A-OLA-T relative to Basic OLA can be expressed as $F L E=\frac{E_{\text {rad (O) }}}{E_{\text {rad (OT) }}}$, and in terms of relatives as FLE $=\{$ (ratio of areas) $\mathrm{x}$ (ratio of minimum node degrees) $\}^{-1}$.

\subsection{Limitations of Analytical Approach}

The minimum node degrees for Basic OLA and OLA-T given by (1) and (2), respectively, hold only for $\gamma=2$, and need to be evaluated for higher path loss exponents. Also, the radii definitions for computing the ratio of areas also depend on $\gamma$. Thus, both the ratios in the expression for FLE (from above) depend on $\gamma$, implying that FLE depends on $\gamma$. The system parameters of interest can be obtained by iteratively solving the aggregate path loss function from a circular disc of radius $r_{0}$ at an arbitrary distance $p>d_{0}$ from the source for $\tau_{l}$ (and $\tau_{u}$ for OLA-T). For an arbitrary choice of $\gamma$, the aggregate path loss function is given by:

$$
f\left(r_{0}, p\right)=\int_{0}^{r_{0}} \int_{0}^{2 \pi}\left[(p-r \cos \theta)^{2}+r \sin \theta^{2}\right]^{\frac{-\gamma}{2}}
$$

for which there are no closed-form solutions when $\gamma>2$, and so it is computed numerically. 
In order to evaluate FLE under the path loss model assumption for higher values of $\gamma$ $(\gamma>2)$, we proceed as follows. First, the minimum node degree, $K_{O, \min }$, for infinite broadcast using Basic OLA is obtained for a disc-shaped network under the continuum assumption. Using Monte-Carlo simulations, we verify these results for random network realizations with finite node densities. Next, the minimum node degree, $K_{O T \text {,min, }}$ which guarantees infinite network broadcast when using OLA-T is obtained for higher path loss exponents. We consider $\gamma=3$ and 4 . For each $\gamma$, the OLA boundaries are computed by solving the above equation numerically for Basic OLA and OLA-T, both operating in their minimum power configurations. Using these results, the FLE achieved by A-OLA-T relative to Basic OLA for each $\gamma$ is obtained. The results along with the details of the simulations are presented in the following sections.

\section{Results and Discussions}

The numerical analysis was performed using Matlab. For the continuum case, 1000 radii definitions (levels) were computed iteratively for different values of $\gamma$ to test for infinite broadcast. We considered $\gamma=2,3$, and 4 , and a range of values for the node degree, $K$. The source power, $P_{s}$ was chosen to be 3 and the decoding threshold, $\tau_{l}$ was 1 . The minimum node degrees for Basic OLA and OLA-T, $K_{O, \min }$ and $K_{O T, \min }$, respectively, corresponded to the values of $\mathrm{K}$ at which the radii stopped increasing, i.e., only a finite portion of the network was reached. Additionally, for OLA-T, each $K_{O T, \min }$ corresponded to a lower bound on RTT, $R_{\text {lower bound. }}$ The simulation framework considered 2000 nodes, randomly and uniformly distributed on a two-dimensional disc of radius 20 distance units with the source node located at the center. The Monte-Carlo simulations were used to obtain the results. A successful network broadcast was defined as the scenario when $99 \%$ of the nodes in the network could decode the message. The Monte-Carlo results were obtained from a simulation of 400 random network realizations. Normalized values were used in each case. The source and relay powers were chosen to be 3 and 0.5 , respectively. The decoding threshold, $\tau_{l} 1$, and the reference distance, $d_{0}$ were assumed to be unity. Nodes in the first level used an $R=5.44$ in $\mathrm{dB}$, for all the trials. Lastly, the minimum node degrees to ensure infinite network broadcast for the two node density cases are within $10 \%$ of each other, thereby validating the continuum assumption and adding confidence to the numerically obtained results.

\subsection{Minimum Node Degree for Basic OLA, $K_{O, \min }$}

The variation of the probability of successful broadcast (PSB) as a function of node 
degree for different path loss exponents, $\gamma$, for Basic OLA is shown in Figure 2. The following values were consider for $\gamma=2,3$, and 4 . The plot shows the simulation to obtain the minimum node degree, $K_{O, \min }$, for a non-coherent OLA-based cooperative broadcast. $K_{O \text {,min }}$ is also evaluated for different network density cases, namely the continuum and the finite density. The results for the continuum case are discussed first. The abscissa is the node degree and the ordinate is the probability of a successful broadcast. The step function that represents the continuum assumption is plotted for each $\gamma$. It can be observed that as the path loss exponent, $\gamma$, increases from 2 to $4, K_{O \text {, min }}$ increases from 1.44 to $\sim 3$ (black curves without symbols). It is noted that the $K_{O, \min }$ for $\quad \gamma=2$ obtained numerically is consistent with [14]. In order to validate the numerical results for the continuum case, we considered random networks with finite node densities to obtain $K_{O \text {,min }}$ for different $\gamma$. As expected, the minimum node degree required for a successful broadcast is slightly higher for the finite node density case, and when $\gamma$ increases from 2 to $4, K_{O \text {, min }}$ increases from $\sim 1.6$ to $\sim 3.2$ for the finite node density case (blue curves). It is noted that the $K_{O \text {,min }}$ for $\gamma=2$ obtained numerically is very close to the theoretical value from [14]. Lastly, the minimum node degrees to ensure infinite network broadcast for the two node density cases are within $10 \%$ of each other, thereby validating the continuum assumption and adding confidence to the numerically obtained results.

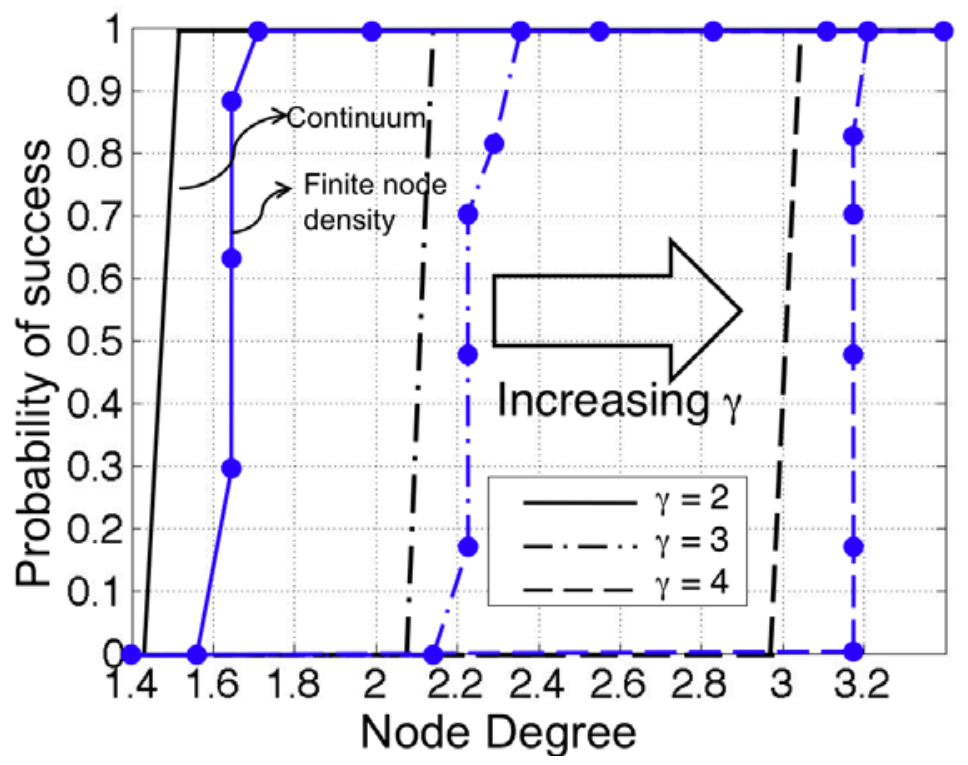

Figure 2. Variation of the probability of successful broadcast (PSB) for Basic OLA for different path loss exponents, $\gamma$. The blue and black curves represent the finite node density and continuum cases, respectively. 


\section{Macrothink}

\subsection{Numerical Lower Bounds on RTT for OLA-T}

Figure 3 shows $R_{\text {lower bound, }}$ in $\mathrm{dB}$, versus the node degree for different path loss exponents, $\gamma=2,3$, and 4, for OLA-T. These results are for the continuum case only. It can be observed

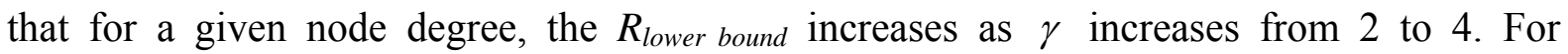
example, for $K=10$, the minimum transmission threshold is $\sim 0.1 \mathrm{~dB}$ for path loss exponent 2 (solid line). However, the minimum transmission threshold is $\sim 1 \mathrm{~dB}$ (dash-dotted line) and $\sim 2.2 \mathrm{~dB}$ (dashed line) for $\gamma=3$ and 4 , respectively. So the value of $R$ for sustained OLA propagations when $\gamma=2$ is insufficient when $\gamma>2$. Alternatively, this implies that higher node degrees are required for operating OLA-T in its minimum power configuration as increases. For example, compared to $\gamma=2$, there is a $20 \%$ increase in the required node degree for infinite network broadcast when $\gamma=4$. It is also remarked that operating at $R_{\text {lower }}$ bound may not be very effective if the precision in the estimate of the SNR is not good enough. All these factors increase the thickness of the OLAs in each hop/energy consumption of OLA-T at higher path loss exponents, thereby affecting the FLE of A-OLA-T. We remark that same numerical lower bounds were obtained for the infinite disc and strip networks for different path loss exponents. This is not surprising since similar conditions for sustained broadcast held for path loss exponent, $\gamma=2$, for both the disc and strip networks using Basic OLA [4], [14] and OLA-T [5], [9]. Lastly, it is remarked that the optimum A-OLA-T performance is achieved when $R_{\text {lower bound }}=R_{\text {upper bound }}$ at $K_{(\mathrm{OT}, \min )}$ [7], [9].

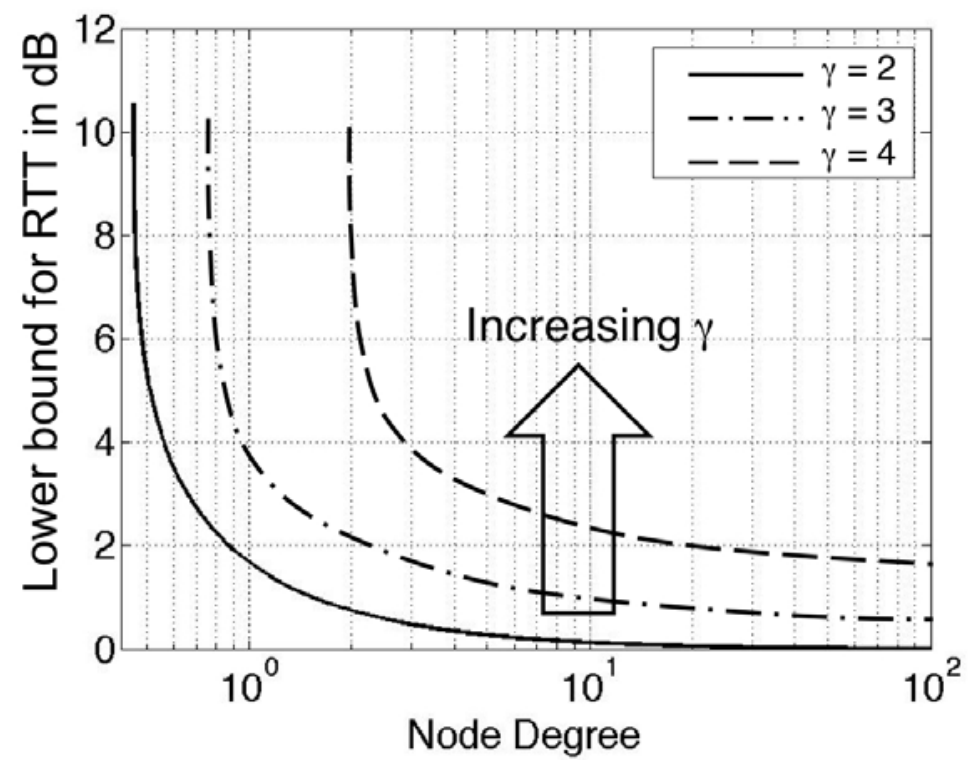

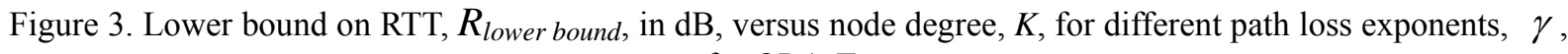
for OLA-T. 


\subsection{Network Lifetime Extension}

Figure 4 shows FLE versus minimum node degree, $K_{(O T, m i n)}$ (on a logarithmic scale), for a disc and strip-shaped network with 1000 levels for different values of. The black (top) and blue (bottom) curves represent the FLE curves for the strip- and disc-shaped networks, respectively.

First, the FLE performance for the disc-shaped network will be discussed. For example, when $\gamma=2$ (solid line), at $K_{(O T, \min )}=10$, FLE is about 1.42 . This means that at their respective lowest energy levels at $K_{(O T, \text { min })}=10$, A-OLA-T extends network life by about $142 \%$ relative to Basic OLA. On the other hand, for $K_{(O T, \min )}=10$ and $=4$ (dashed line), the FLE is about 1.34, meaning that A-OLA-T offers a network life extension of about $134 \%$ relative to Basic OLA, both protocols operating in their minimum power configurations. It is noted that FLE increases with $K_{(O T, \min )}$ and attains a maximum of about $142 \%$ (for $\gamma=3$ ) and about $137 \%$ (for $\gamma=4$ ). Next, consider the curves for the strip-shaped network. When $\gamma=2$ (solid line), at $K_{(O T, \min )}=10, \mathrm{FLE}$ is about 2.50 . This means that at their respective lowest energy levels at $K_{(\text {OT,min })}=10$, A-OLA-T extends network life by $250 \%$ relative to Basic OLA, more than that obtained for a disc-shaped network, which should not be surprising. On the other hand, for $K_{(O T, \min )}=10$ and $\gamma=4$ (dashed line), the FLE is about 1.80, meaning that A-OLA-T extends network life by $180 \%$ relative to Basic OLA, both protocols operating in their minimum power configurations. It is noted that FLE increases with $K_{(O T, \min )}$ and attains a maximum of about $238 \%$ (for $\gamma=3$ ) and about $200 \%$ (for $\gamma=4$ ).

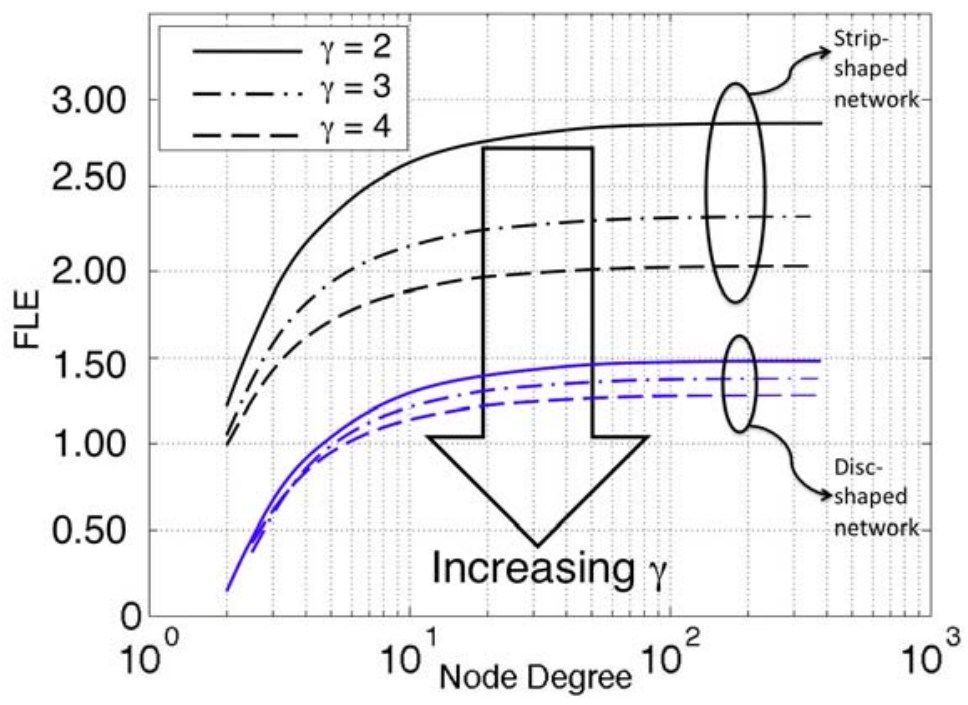

Figure 4. Variation of FLE with the minimum node degree for a disc- and strip-shaped network with 1000 levels for different path loss exponents. 


\section{Concluding Remarks}

It is concluded that the A-OLA-T broadcasting strategy is still an energy-efficient option compared to Basic OLA, especially for successive network broadcasts in a static network.

However, it was observed that increasing the path loss exponent, $\gamma$ from 2 to 4 required more hops to achieve network broadcast with slightly thicker OLAs implying increased node participation in each hop. Increasing $\gamma$ increased the minimum relay transmit power per unit area, $\operatorname{Pr}(\mathrm{OT}, \mathrm{min})$ (and subsequently, the minimum node degree, $\mathrm{K}(\mathrm{OT}, \mathrm{min})$ ) and the lower bound on RTT, Rlower bound for sustained network broadcast using OLA-T. Therefore, the fraction of transmit energy saved by OLA-T relative to Basic OLA decreased at high path loss exponents. To summarize, contrary to our initial hypothesis that using only the border nodes in the case of OLA-T will widen the gap in energy consumption between Basic OLA and OLA-T, it is found that the OLA-T energy savings, and consequently, the A-OLA-T network life extensions while operating at a higher path loss $\left(\gamma_{>}\right)$are lower. This implies that for very lossy channels $(\gamma>4)$, the energy savings.

\section{References}

[1] A. Fort, J. Ryckaert, C. Desset, P. De Doncker, P. Wambacq, and L. Van Biesen, "Ultra-wideband channel model for communication around the human body," IEEE J. Sel. Areas Commun., vol. 24, pp. 927-933, Apr. 2006. http://dx.doi.org/10.1109/JSAC.2005.863885

[2] A. Sendonaris, E. Erkip, and B. Aazhang, "User cooperation - Part I: System description, Part II: Implmentation aspects and performance analysis," IEEE Trans. Commun., vol. 51, no. 11, pp. 1927-1948, Nov. 2003. http://dx.doi.org/10.1109/TCOMM.2003.818096

[3] J. N. Laneman, D. Tse, and G. W. Wornell, "Cooperative diversity in wireless networks: Efficient protocols and outage behavior,'IEEE Trans. Inf. Theory, vol. 50, no. 12, pp. 3063-3080, Dec. 2004. http://dx.doi.org/10.1109/TIT.2004.838089

[4] Y. W. Hong and A. Scaglione, "Energy-efficient broadcasting with cooperative transmissions in wireless sensor networks," IEEE Trans. Wireless Commun., vol. 5, no. 10, pp. 2844-2855, Oct. 2006. http://dx.doi.org/10.1109/TWC.2006.04608

[5] A. Kailas, L. Thanayankizil, and M. A. Ingram, "A simple cooperative transmission protocol for energy-efficient broadcasting over multi-hop wireless networks," KICS/IEEE J. of Commun. and Netw., vol. 10, no. 2, pp. 213-220, June 2008. http://dx.doi.org/10.1109/SENSORCOMM.2007.4394976

[6] B. Sirkeci-Mergen and A. Scaglione, "On the power efficiency of cooperative broadcast in dense wireless networks," IEEE J. Sel. Areas Commun., vol. 25, no. 2, pp. 497-507, Feb. 2007. http://dx.doi.org/10.1109/JSAC.2007.070223

[7] A. Kailas and M. A. Ingram, "Alternating opportunistic large arrays in broadcasting for network life extension," IEEE Trans. Wireless Commun., vol. 6, no. 8, pp. 2831-2835, June 2009. http://dx.doi.org/10.1109/TWC.2009.080729 


\section{Macrothink}

[8] Y. J. Chang, M. A. Ingram, and R. S. Frazier, "Cluster transmission time synchronization for cooperative transmission using software defined radio," Proc. IEEE CoCoNet Workshop, ICC, May 2010. http://dx.doi.org/10.1109/ICCW.2010.5503961

[9] A. Kailas and M. A. Ingram, "Analysis of a simple recruiting method for cooperative routes and strip networks," IEEE Trans. Wireless Commun., vol. 9 , no. 8, pp. 2415-2419, Aug. 2010. http://dx.doi.org/10.1109/TWC.2010.062910.091175

[10] S. Slijepcevic and M. Potkonjak, "Power efficient organization of wireless sensor networks," Proc. ICC, June 2001, pp. 472-476. http://dx.doi.org/10.1109/ICC.2001.936985

[11] M. Cardei and D. -Z. Du, "Improving wireless sensor network life through power aware organization," ACM Wireless Netw., vol. 11, no. 3, pp. 333-340, 2005. http://dx.doi.org/10.1007/s11276-005-6615-6

[12] T. N. Nguyen and D. T. Huynh,"Extending sensor networks life through energy efficient organization," Proc. WASA, Aug. 2007, pp. 207-212. http://dx.doi.org/10.1109/WASA.2007.7

[13] G. Trajcevski, O. Ghica , P. Scheuermann, R. Tamassia, and I. F. Cruz, "Alternating multiple tributaries+deltas,” Proc. DMSN, 2008, pp. 28-34. http://dx.doi.org/10.1145/1402050.1402057

[14] B. Sirkeci-Mergen, A. Scaglione, G. Mergen, "Asymptotic analysis of multi-stage cooperative broadcast in wireless networks," EEE Trans. Inf. Theory and IEEE/ACM Trans. Netw., vol. 52, no. 6, pp. 2531-2550, June 2006. http://dx.doi.org/10.1109/TIT.2006.874514

[15] R. Mudumbai, G. Barriac, and U. Madhow, "Spread-spectrum techniques for distributed space-time communication in sensor networks," Proc. Asilomar Conference Signals, Systems and Computers, pp. 908-912, Nov. 2004. http://dx.doi.org/10.1109/ACSSC.2004.1399270

\section{Copyright Disclaimer}

Copyright reserved by the author(s).

This article is an open-access article distributed under the terms and conditions of the Creative Commons Attribution license (http://creativecommons.org/licenses/by/3.0/). 